\title{
Un proyecto de intervención para el desarrollo del talento en Matemáticas para niñas con edades entre 12 y 16 años
}

Resumen: La escasa presencia de las mujeres en Olimpiadas de Matemáticas parece ser una constante en México, Latinoamérica e incluso en occidente. Esta situación se ha obviado, y hasta cierto modo naturalizado, por las instituciones $\mathrm{y}$ personas implicadas. Analizamos como esto se vive en el estado de Tamaulipas en México, enmarcada por las políticas públicas y la influencia de la Olimpiada propuesta por la Sociedad Matemática Mexicana. Describiremos las acciones que hemos llevado a cabo para fomentar la participación de más niñas, pero también para apoyar el desarrollo del potencial, motivación e intereses de quienes ya participan en ellas. Por medio de un análisis descriptivo de su trayectoria en el concurso encontramos que pesar de tener un alto desempeño, es muy poco probable que elijan una carrera relacionada con Matemáticas. Factores como el profesorado y la familia tienen una influencia importante, pero también la dinámica de este tipo de eventos.

Palabras clave: Talento. Género. Olimpiadas de Matemáticas.

\section{An intervention project for the development of talent in Mathematics}

\section{for girls with between the ages of 12 and 16}

Abstract: The low presence of women on Mathematics Olympics sems to be a constant in Mexico, Latin America and all the world. This situation was highlighted and, to a certain extent, naturalized by the institutions and people involved. We analyze how this is lived at Tamaulipas in Mexico, framed by public politics and the influence of the Olympiad proposal by the Mexican Society of Mathematics. We will describe the actions that we have executed to encourage the participation of more girls, but also to support their potential development, motivation, and interest of those who already participate in them. Through a descriptive analysis of their career in the competition, we found that despite having a high performance, it is very unlikely that they will choose a career related to Mathematics. Factors such as the teachers and the family have an important influence, but also the dynamics of this type of events.

Keywords: Talent. Gender. Mathematics Olympics.

\section{Um projeto de intervenção para o desenvolvimento de talentos em Matemática para meninas de 12 a 16 anos}

Resumo: A baixa presença de mulheres nas Olimpíadas de Matemática é uma constante no México, na América Latina e até no Ocidente. Esta situação foi evidenciada e, em 
certo ponto, naturalizada pelas instituições e pessoas implicadas. Analisamos como isso é vivido no estado de Tamaulipas, no México, emoldurado por políticas públicas e a influência da Olimpíada proposta pela Sociedade Mexicana de Matemática. Descreveremos as ações que realizamos para incentivar a participação de mais meninas mas, também, para apoiar o potencial desenvolvimento, motivação e interesse de quem já participa delas. Por meio de uma análise descritiva de sua trajetória no concurso, constatamos que apesar de terem um alto desempenho, é muito improvável que optem por uma carreira relacionada à Matemática. Fatores como os professores e a família têm uma influência importante, mas também a dinâmica desse tipo de eventos.

Palavras-clave: Talento. Gênero. Olimpíadas de Matemática.

\section{Introducción}

Desde la década de los 1980, diversas investigaciones se han ocupado de analizar los fenómenos relacionados al género y a las habilidades sobresalientes en matemáticas (DOMÍNGUEZ, 2002; LEE y SRIRAMAN, 2011; FARFÁN y SIMÓN, 2016; BLACK et al., 2017). Situaciones como la escasa representación de las mujeres en el campo de las Ciencias Exactas; la baja proporción de ellas que forman parte de programas dirigidos a la atención de las personas altamente dotadas en Matemáticas; así como los intereses, motivaciones y emociones que experimentan las jóvenes, han sido analizadas.

Se ha identificado en pruebas estandarizadas como Programa Internacional de Evaluación de Estudiantes (PISA) a nivel internacional y Planea en México, que las fracciones de la población que no tienen éxito en las evaluaciones nacionales e internacionales de Matemáticas coinciden con ciertos grupos étnicos, sectores socioeconómicos $\mathrm{y}$, recientemente identificado, las mujeres como grupo social (MÁRQUEZ, 2017; SIMÓN, 2018). Justamente estos sectores de la población son los que están menos representados en los grupos de individuos identificados con capacidades sobresalientes, principalmente en matemáticas. Tal como lo podemos observar en las recientes selecciones nacionales mexicanas para olimpiadas, en las cuáles las mujeres son una marcada minoría. Por mencionar uno de los concursos en los que México participa, la Olimpiada Internacional de Matemáticas, de 2015 a 2020 la selección enviada solo ha incluido a dos mujeres y una de ellas participó en dos años consecutivos. Un fenómeno muy similar se ha identificado en la población Tamaulipeca, pues el número de estudiantes participantes en programas de atención a las habilidades sobresalientes en Matemáticas y en olimpiadas disminuye considerablemente con la edad.

En conjunto con redes e instituciones interesadas en el desarrollo del potencial de 
las mujeres — Red de Ciencia, Tecnología y Género; Consejo Nacional de Ciencia y Tecnología - planteamos una propuesta dirigida a las niñas y adolescentes de Tamaulipas que han mostrado interés o un alto potencial hacia Matemáticas. Con el objetivo de apoyar su participación en programas para el desarrollo de sus habilidades en matemáticas o en áreas que la toman como una herramienta - principalmente las denominadas STEM; Ciencia, Tecnología, Ingeniería y Matemáticas. Dicho proyecto consistió en ofrecerles un espacio en el cuál pudieran desarrollar sus habilidades, considerando los principales aspectos que las investigaciones en género y desarrollo del talento en Matemáticas han identificado. Esto lo realizamos principalmente a través de conferencias y talleres.

Cada año el estado de Tamaulipas (México) participa en varios concursos de Matemáticas. La Universidad Autónoma de Tamaulipas (UAT) contribuye con la organización de dos de estos eventos de Olimpiadas: la Olimpiada para Educación Media Superior (15 a 17 años) y la de Básico (9-14). Se presentan datos obtenidos en 2018 para enmarcar los motivos que dan origen a este proyecto. El número de participantes en la primera de ellas fue aproximadamente de 2500 y 1500 para la segunda. Para el caso de la olimpiada a Nivel Básico la participación de las niñas comenzó con un 49\% y a través de un proceso de eliminación - con pruebas tradicionales de olimpiadas - se obtuvo una selección nacional que participó en diferentes eventos nacionales, en la cual solo 6 de 17 estudiantes seleccionados fueron mujeres (35\%). Para el caso de medio superior la participación de las niñas en su primera etapa fue de $45 \%$ y finalizó con 2 niñas dentro de una selección de 18 (11\%).

El proyecto que se reporta en este artículo tiene como objetivo apoyar la permanencia e incorporación de más mujeres en este tipo de actividades, así como trabajar en el desarrollo de sus intereses y autoconcepto como sobresalientes en matemáticas, para lograr el aprovechamiento del potencial que han mostrado tener.

Presentamos una parte del proyecto, la cual se enfoca en promover la inclusión de más niñas en los cursos de Matemáticas avanzadas y en talleres científicos, buscando contribuir al fomento y desarrollo de vocaciones. Creemos que de este modo podremos evitar que las niñas y adolescentes talentosas desistan de participar en actividades que desarrollan su potencial en Matemáticas, o sean excluidas por un proceso de selección que no considera las particularidades de quienes participan. Aumentando así, a largo 
plazo, la proporción de mujeres Tamaulipecas que optan por una carrera en Matemáticas, pero también en otras áreas STEM.

El proyecto apoyó y acompañó a 17 niñas — aproximadamente 50\% de la selección estatal - en su proceso de preparación para participar en la olimpiada dirigida a nivel medio superior, pero también se llevaron a cabo talleres de forma paralela, dirigidos exclusivamente a ellas. Se espera que este tipo de acciones tengan a la larga un impacto mayor entre las futuras participantes de todo el estado y todos los niveles educativos.

\section{Equidad, capacidades sobresalientes y género}

En México la denominada educación especial considera tanto a la población con alguna discapacidad como al estudiantado que sobresale en algún aspecto de su formación académica (MÉXICO, 2006).

La Secretaría de Educación Pública de México, ha definido desde 2006 al estudiantado con aptitudes sobresalientes como, aquellas personas capaces de destacar significativamente del grupo social y educativo al que pertenecen en uno más de los siguientes aspectos: científico-tecnológico, artístico o de acción motriz. Considera que, al presentar necesidades específicas, requieren de un contexto facilitador que les permita desarrollar sus capacidades personales y satisfacer sus necesidades e intereses para su propio beneficio y el de la sociedad.

Para lograr la atención integral de este grupo se plantearon dos estrategias de intervención: el enriquecimiento y la aceleración. El primero consiste en una serie de acciones planeadas por los docentes de educación regular en conjunto con los de educación especial y las autoridades educativas involucradas. De manera estratégica se toma en cuenta los intereses, potencialidades y debilidades del estudiantado identificado con capacidades sobresalientes. El segundo considera la movilidad curricular al ritmo que el estudiantado necesite y puede consistir en la admisión temprana a algún nivel educativo o la omisión de algún grado escolar.

En 2006 estas propuestas de intervención se pusieron en juego en toda la República Mexicana, pero poco se conoce de sus resultados. Covarrubias y Marín (2015) reportan que solo en el estado de Hidalgo y en Chihuahua se han dado a conocer las 
evaluaciones de las intervenciones llevadas a cabo en los respectivos estados. Estos autores concluyeron que teóricamente la propuesta de intervención estaba bien sustentada, pero operativamente no había logrado los resultados esperados. Los principales problemas se habían presentado en los mecanismos de capacitación del profesorado y por lo tanto esto tenía una repercusión directa en la intervención con el estudiantado por parte del personal de educación regular y especial, traduciéndose en un trabajo no coordinado entre estas dos figuras. Como resultado, no se cubrieron las necesidades educativas del estudiantado con aptitudes sobresalientes. El mismo trabajo reporta que no existen evidencias cualitativas que atestigüen los avances en términos de calidad de resultados y se reflejen en el desarrollo del potencial de las y los alumnos identificados y registrados estadísticamente. Pudimos constatar que en los documentos de trabajo que aparecen en la página web del programa nacional no se presenta ninguno que se refiera a la evaluación del impacto del programa en el desarrollo del potencial del estudiantado. Una historia parecida a esta se ha dado con programas anteriores a este. Como el programa para la atención de las Capacidades y Aptitudes Sobresalientes de 1985 (SIMÓN, 2009).

Existen otro tipo de propuestas de detección y atención que se llevan a cabo según surjan las convocatorias a lo largo del año y estas son las olimpiadas de conocimientos, desde las generales hasta las relacionadas con alguna asignatura. Para estas la práctica tradicional consiste en que los docentes proponen a un grupo de estudiantes y les apoyan académicamente a lo largo de todas las etapas que considere dicho concurso, ya sea estatal, nacional o internacional.

Este es el caso de las Olimpiadas de Matemáticas. Si bien, estas pueden llevarse a cabo por las autoridades educativas estatales, el evento nacional que dicta las pautas de estos es en la mayoría de los casos el organizado por la Sociedad Matemática Mexicana (SMM) la Olimpiada Mexicana de Matemáticas (OMM), el cuál anteriormente se realizaba solo para el nivel medio superior y ahora se ha ampliado a Secundaria y Primaria. La etapa nacional de la OMM para el nivel medio superior se realiza durante noviembre y para ello cada uno de los estados participantes trabaja durante todo el año para tener una selección de estudiantes que les represente. Aunque la etapa nacional se lleva a cabo con los recursos gestionados por parte de la SMM, todo el proceso realizado durante las etapas estatales queda en manos de los llamados comités de la OMM. Quienes 
los integran pueden o no pertenecer formalmente a una institución educativa (OMM, 2021), ya que este no es un requisito que se especifique en el procedimiento de selección de estos. Son estas personas quienes se encargan de organizar todo lo relacionado con la selección del grupo que les representará a nivel nacional, desde eventos de eliminatoria hasta la obtención de recursos económicos para que puedan asistir al lugar dónde se lleva a cabo la prueba final.

Poco se conoce sobre la organización al interior de las instituciones educativas para apoyar la participación del estudiantado. Es conocido que, apoyan económicamente a quien participa y a su acompañante para viajar al lugar donde se lleve a cabo la etapa en la que participan, de tal manera que es el recurso económico institucional lo que permite llevar a cabo el evento, el cual se da gracias a la encomienda que hace a las escuelas la Subsecretaría de Educación Media Superior del Estado.

Conocer y caracterizar la organización institucional, entre docentes, padres, madres y el estudiantado es un tema pendiente para esta investigación. Creemos que así, podremos identificar otros aspectos que puedan influenciar la participación de las jóvenes mujeres y planeamos realizarlo en un futuro próximo.

\section{Identificación de capacidades sobresalientes}

Los modelos de identificación y atención a las personas con capacidades sobresalientes han tenido una gran evolución. Desde los inicios del siglo XX cuando se propuso la medición de la inteligencia por medio de una prueba estandarizada, que medía el cociente intelectual o índice de inteligencia (CI). Hasta la consideración de factores genéticos, culturales, personales y motivacionales.

Todos estos estudios han llevado a la diferenciación del talento, la genialidad y la superdotación - entre otros términos regularmente utilizados como sinónimos), lo cual posibilita un tratamiento educativo diferenciado que responda a los intereses o necesidades educativas de unos y otros atendiendo a sus características (SIMÓN, 2009). Esto ha llevado a transitar de una concepción general a una que pone mayor énfasis a los talentos específicos. Tomemos como ejemplo al modelo de los Tres Anillos de Renzulli y el de Inteligencias Múltiples de Gardner por ser los más conocidos y utilizados dentro de las políticas de atención e identificación propuestas en México. 
Si bien nuestra postura no coincide del todo con estos modelos, sí consideramos que la habilidad humana no puede ser medida únicamente por medio de una prueba estandarizada, pues está conformada por una diversidad de elementos como destrezas aprendidas, motivaciones, aptitudes y disposiciones (personales y contextuales) que llevan a una persona a desarrollar su potencial en cierta medida.

Como ya habíamos mencionado, la evolución conceptual y técnica de la medición de la inteligencia ha tomado como elemento de discusión la distinción entre los diversos términos asociados a las personas con capacidades sobresalientes: precoz, genio, superdotado, sobredotado o talento. Desde nuestra postura distinguimos a la de talento, dado que su conceptualización se ha desarrollado a la par de los más recientes modelos teóricos. Dicho término, surge de entre estos modelos como un cambio de paradigma, que lo dota de un carácter flexible, dinámico e incluyente. Bajo la perspectiva socioepistemológica, desde la cual se considera que el conocimiento se construye socialmente, consideramos al talento como desarrollable en su interacción con el medio sociocultural - familia, escuela, sociedad - a la luz de un saber matemático transversal y funcional. Y estará caracterizado por las herramientas del pensamiento matemático que el individuo domine y de las cuáles haga uso, que le permitan tomar una decisión respecto a la manera de abordar un problema, argumentarlo y resolverlo (CANCHÉ y FARFÁN, 2017). Por lo tanto, es vital estudiar al talento en las interacciones con los procesos y ámbitos en los que se desarrolla.

La mayoría de las propuestas contemporáneas de identificación y atención de las capacidades sobresalientes, consideran como un elemento importante la selección del profesorado (SIMÓN, 2009) pues serán quienes reconozcan en el estudiantado un potencial que merece ser desarrollado y apoyado mediante su participación en actividades de enriquecimiento y eventos especiales como las olimpiadas.

En México, en el nivel medio superior el porcentaje de profesores supera al de profesoras con el 51.8\%, y cuando se trata de la asignatura de Matemáticas este porcentaje aumenta debido a que en su mayoría el profesorado que imparte las asignaturas de Matemáticas ha egresado de alguna carrera de áreas tradicionalmente masculinas como las Ingenierías. Dado que el comportamiento de niños, niñas y jóvenes está influenciado por el comportamiento de aquellos y aquellas con quienes socializa (FLORES, 2007), será menos probable que las estudiantes mujeres encuentren profesoras con quien 
identificarse y por lo tanto visualizarse dentro de un programa, formal o informal, de atención a sobresalientes en matemáticas.

Por otro lado, está la nominación por parte del profesorado, es decir, son quienes seleccionan y convocan al estudiantado que formará parte del grupo que se preparará para las Olimpiadas de Matemáticas u otra asignatura.

Otras investigaciones, en otras naciones, han mostrado que es menos probable que las niñas se unan a equipos de Matemáticas o que sean apoyadas para ello y por lo tanto están menos representadas en estos grupos (ELLISON y SWANSON, 2010; LEE y SRIRAMAN, 2012). Las razones para esto son diversas y dependen del contexto nacional. Vale la pena preguntarse sobre las dinámicas que llevan a que esto suceda en México, en especial en el norte del país.

\section{Mujeres en Olimpiadas de Matemáticas}

Consideramos a las adolescentes que forman parte de este proyecto como talentosas, ya que son reconocidas por su familia, su entorno educativo y social como destacadas en Matemáticas. Y lo refrendan a través de su participación, avance y permanencia en este tipo de concursos. Sin embargo, como hemos visto, su presencia se reduce conforme avanzan en las etapas de este tipo de concursos.

Es la SMM quien año con año abre la convocatoria para el concurso nacional para estudiantes preuniversitarios. Es a través de este medio de selección que alrededor de 16 estudiantes de todo el país con las más altas puntuaciones podrán participar en entrenamientos especiales durante varios meses. De este grupo se seleccionará a los equipos que formarán parte de diferentes olimpiadas internacionales: la Internacional, la Iberoamericana, la de Centroamérica y el Caribe y la correspondiente a la Cuenca del Pacífico. En los últimos años mediante este mismo concurso también se elige al equipo que participará en la Olimpiada Europea Femenil de Matemáticas (OMM, 2021).

Las personas que integrarán estos equipos tienen que cumplir como requisito obtener los mejores resultados del proceso selectivo que se lleva a cabo dentro de estos entrenamientos. El cual consiste en una serie de exámenes que van descartando estudiantes en varios periodos establecidos. Por lo tanto, este proceso está principalmente fundado en la competencia entre sus integrantes más que en la colaboración y apoyo 
mutuos, estos últimos son valores que actualmente se trata de promover en todos los campos científicos.

La Olimpiada Internacional es considerada la más importante en el nivel medio superior. Los datos desglosados por sexo correspondientes a los equipos mexicanos que año con año participan en esta competencia nos dan una muestra de la presencia de las mujeres en este tipo de evento.

Tabla 1: Participantes en la Olimpiada Internacional de Matemáticas, distinción por sexo

\begin{tabular}{c|c|c}
\hline Año & \# Hombres & \# Mujeres \\
\hline 2015 & 6 & 0 \\
\hline 2016 & 5 & 1 \\
\hline 2017 & 6 & 0 \\
\hline 2018 & 6 & 0 \\
\hline 2019 & 5 & 1 \\
\hline 2020 & 5 & 1 \\
\hline \multicolumn{3}{|c}{ Fuente: Elaboración Propia }
\end{tabular}

Es importante mencionar que esta tabla ha sido elaborada con la información que aparece en los diferentes medios de comunicación - noticieros, periódicos, revistas y blogs de internet —, debido a que la OMM no tiene en su sitio de internet un espacio destinado a ofrecer este tipo de datos.

\section{Investigaciones en género, talento y Matemáticas}

Es bien sabido, pero se le da poca importancia, que el papel de las mujeres dentro de las Ciencias siempre ha sido dejado en un segundo lugar. Evidencia de este fenómeno se ha dado desde varias investigaciones. Mencionaré al efecto Matilda por la relevancia que ha tenido en la actualidad. Este fenómeno va más allá de no considerar la participación de las mujeres en el desarrollo científico a el robo, plagio o atribución del trabajo de las mujeres a hombres cercanos a ellas, como sus mentores, esposos, padres o hermanos (MARTINEZ, 2014).

En un primer momento, las actividades asignadas socialmente a las mujeres, las cuáles se quedaban en el ámbito de lo privado, no fueron un impedimento para que muchas de ellas dedicaran su conocimiento y esfuerzo a desarrollar trabajo científico. Aún con la imposibilidad de ingresar a las universidades o pertenecer a las sociedades 
científicas ellas siguieron haciendo importantes contribuciones. Fue la matematización de las ciencias y el establecimiento de esta como un campo del conocimiento reservado solo para unos cuantos lo que llevó a que el trabajo de las mujeres fuera devaluado y, por lo tanto, se les consideró doblemente negadas para acceder a ese tipo de conocimiento (FARFÁN y SIMÓN, 2016). Prueba de ello la podemos observar en los datos que acabamos de presentar. Aún en pleno siglo XXI las mujeres siguen sin ser reconocidas y apoyadas en el desarrollo de su potencial.

El ingreso de las mujeres a una educación formal y mixta ha mostrado grandes avances en cuanto al número de mujeres que estudian una carrera o un posgrado (SUBIRATS, 2010). En México, incluso en algunas generaciones las mujeres llegan a superar a los varones con más del 50\% de la matrícula en las licenciaturas. Sin embargo, se mantiene una segregación horizontal con un mayor número de mujeres en carreras tradicionalmente asociadas a lo femenino - educación y humanidades; ciencias de la salud; ciencias sociales y administrativas (SIMÓN, 2018). Y una segregación vertical que tiene a las mujeres lejos de los lugares de más alto reconocimiento (Sistema Nacional de Investigación y Academias de Ciencias) y de decisión (direcciones y rectorías de universidades y centros de investigación) (BLAZQUEZ, BUSTOS y FERNÁNDEZ, 2012) dentro de las áreas científicas. Una vez más esto es evidencia de que como sociedad nos perdemos del talento de las mujeres.

Una de las instituciones más influyentes en la transmisión y perpetuación de los roles de género es la escuela. Si bien ya no tenemos una escuela segregada por sexo como sucedía durante el gobierno de Benito Juárez o Porfirio Díaz, las cuáles se distinguían por su currículum diferenciado y una inversión más baja para las escuelas de mujeres (GONZÁLEZ, 2009). Los procesos de socialización en las escuelas reproducen la clásica construcción de roles derivados de la división sexual del trabajo, determinando trayectorias, comportamientos, actitudes, expectativas y decisiones vocacionales para mujeres y hombres. Esta situación se cristaliza en el acceso diferenciado a los bienes simbólicos y materiales, limitando el acceso equitativo a las oportunidades de desarrollo, especialmente para ellas (POBLETE, 2011).

Investigaciones de larga tradición como las realizadas por Subirats (2010) han constatado que el saber escolar sigue transmitiendo una cultura androcéntrica, que 
naturaliza los comportamientos competitivos y violentos de los chicos dejando de lado los saberes y valores tradicionalmente femeninos. Donde actitudes como la empatía y la entrega son menos apreciadas y actitudes masculinas en las chicas son poco valoradas e incluso tomadas como negativas. Ella identifica que a pesar de los avances en cuando al acceso universal a la educación básica no se ha conseguido (a nivel internacional) un uso equilibrado de los espacios, los tiempos, ni un cambio suficientemente claro en lenguaje, y deberemos agregar, de la atención recibida por ellas y ellos de parte del profesorado. Una situación que creemos se vería intensificada en espacios donde es muy marcada la mayoría masculina tanto en el estudiantado como en el profesorado. Un aspecto que a través de nuestra propuesta hemos tratado de trastocar, ofreciendo espacios para ellas en los que puedan interactuar de una forma más libre, así como equilibrando la participación de mujeres y hombres (estudiantes y docentes). Si bien, este tipo de propuestas son criticadas por estar alejadas de la realidad en la que la humanidad se desarrolla, la necesidad de este tipo de espacios surge ante la falta de verdaderos modelos de coeducación, en el sentido de Subirats (2010). En los cuáles se ponga en el mismo sentido de importancia tanto a niñas como a niños y les permitan desarrollarse con independencia de su sexo.

\section{Modelo de desarrollo del talento}

Desde la perspectiva socioepistemológica, teoría que guía nuestras reflexiones, se propone trastocar a la Matemática escolar de tal manera que el problema educativo no sea la aprehensión de objetos abstractos, sino la democratización del aprendizaje. Es decir, que la ciudadanía disfrute y participe de la cultura matemática enraizada en sus propias vidas (CANTORAL, MONTIEL y REYES, 2014). Objetivos muy parecidos a los que se plantean las Olimpíadas de Matemáticas en México, en palabras de Isabel Hubard, la principal entrenadora del equipo que se envía a la Olimpiada Europea Femenil (UNAM, 2019), "difundir las matemáticas creativas, el razonamiento matemático, la resolución de problemas, en los niños y jóvenes del país, es un poco como tratar de quitar esta imagen negativa que tenemos de las Matemáticas”.

Los datos que hemos mostrado y la literatura universal muestran que las mujeres no tienen la oportunidad de participar en espacios, tanto escolares como extraescolares, en los cuales puedan potenciar su interés y habilidades en matemáticas (LEE y 
SRIRAMAN, 2012). Al tiempo que existen concepciones de la inteligencia o del talento matemático que impiden la democratización del aprendizaje, al excluir a ciertos grupos sociales — bajos recursos económicos, mujeres, discapacidad — de la posibilidad de desarrollar al máximo su potencial.

Una de las posturas contemporáneas en la investigación es la que atribuye a la influencia de los aspectos socioculturales de género el bajo rendimiento en las pruebas estandarizadas o en el logro en Matemáticas, al tener una fuerte influencia en el desarrollo del autoconcepto, motivación e intereses de las niñas y jóvenes respecto a matemáticas u otras áreas STEM.

En investigaciones previas hemos propuesto un modelo de análisis del desarrollo del talento que tiene sus cimientos en todo lo expuesto anteriormente (FARFÁn y SIMÓN, 2016). Por un lado, considera a los métodos actuales para evaluar la habilidad matemática, los cuáles suelen ser pruebas estandarizadas o aquellas enfocadas en la Matemática para olimpiadas. Por lo tanto, es importante considerar, ¿Cuál es la concepción de talento matemático detrás de estas pruebas? ¿Cuál es la concepción de talento matemático que impera en la sociedad y cómo está mediada por la influencia de aspectos socioculturales asociados al género? Y por supuesto, ¿Cómo se ve reflejado en el desarrollo del potencial de las mujeres?

Dicho modelo considera que es a través del análisis de la interacción de tres elementos: Matemáticas, talento y género, que podemos realizar un análisis socioepistemológico del talento en Matemáticas desde una perspectiva de género (Figura 1), el cuál toma en cuenta principalmente al individuo como constructor de conocimiento dentro de un entorno sociocultural. De este modo, las relaciones que establezca con la Matemática estarán en función de éste, dentro del cual sus experiencias se encuentran permeadas por el sexo al que pertenece, dadas las atribuciones sociales que se han dado a mujeres y hombres (FARFÁN y SIMÓN, 2016). 


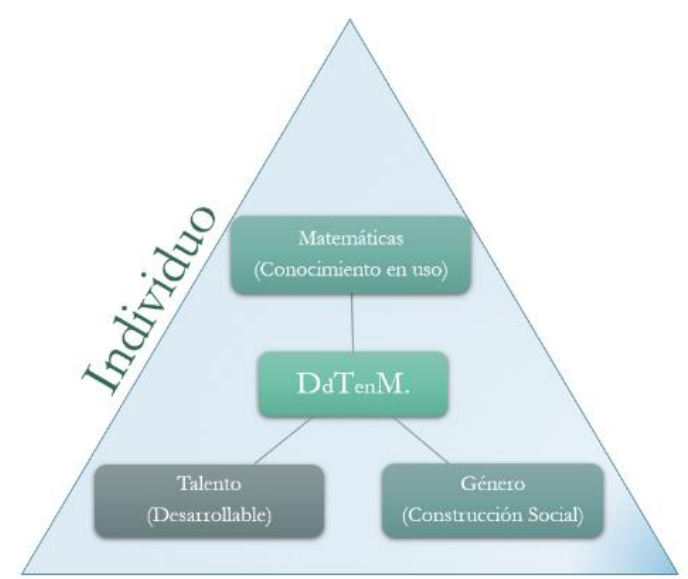

Figura 1: Modelo de análisis para el desarrollo del talento en matemáticas

(FARFÁN y SIMÓN, 2016, p. 187)

Este modelo no tiene como objetivo principal dictaminar quien posee talento matemático, sino que nos da las herramientas para analizar cómo se ha desarrollado, la forma en la que ponen uso su conocimiento y también cómo una persona se constituye a sí misma en relación con la matemática. Y, por lo tanto, nos brinda las herramientas para intervenir en los momentos y la forma necesarios según nuestra población.

\section{Propuesta de atención al desarrollo del talento en Matemáticas para adolescentes tamaulipecas}

Este proyecto tiene como objetivo lograr a largo plazo la permanencia e incorporación de más niñas en las olimpiadas de Matemáticas realizadas en Tamaulipas, pero a su vez, también trabajar en el desarrollo de sus intereses y autoconcepto. Es por lo que en un inicio se planteó como objetivo integrar a una mayor cantidad de niñas en los cursos y entrenamientos que se llevan a cabo para la selección de los equipos de Tamaulipas que participarán en diferentes eventos nacionales. A estos entrenamientos asiste una media de 30 estudiantes y en 2018 solo se integraron 8 mujeres de una selección de $32(25 \%)$ que participó en el nivel medio superior. Pero será a través de la investigación paralela al proyecto que demos evidencia de cuáles son los factores que obstaculizan la participación de estas niñas en estos eventos, los cuáles pareciera las excluyen en cuanto avanzan en edad y en las diversas etapas del concurso. Diversidad de investigaciones han demostrado que no se debe a la habilidad o inteligencia de las mujeres, sino a diversos factores asociados al trato de género y a la creencia de que las matemáticas son un área masculina.

Se realizaron las gestiones necesarias para que 15 niñas (50\% de la selección 
estatal) pudieran participar de dichos entrenamientos (con edades entre 11 y 17 años). Pero también se buscó su participación en diversos talleres y conferencias que se desarrollaron de forma paralela. Estos tuvieron como objetivo influir en su autoconcepto como talentosas en Matemáticas a través de mostrar una visión diferente sobre la construcción de conocimiento matemático e integrando a más mujeres como entrenadoras y talleristas. Se realizó un seguimiento a partir de tres aspectos:

- Su historia de vida, por medio de cuestionarios, entrevistas y seguimiento académico dentro de las olimpiadas.

- Desarrollo del pensamiento matemático por medio del análisis de su desempeño en pruebas y cursos de preparación para olimpiadas durante las emisiones en las que hayan participado.

- Desarrollo de su autoconcepto como talentosas. A través del análisis de su participación en actividades paralelas a las de olimpiada que tienen como objetivo motivarlas e interesarlas en la ciencia. Tales como: talleres con científicas, participación de entrenadoras mujeres y aumentar la presencia de mujeres en el equipo que forma parte de los entrenamientos.

Este artículo reporta los hallazgos del primer aspecto pues consideramos este como el punto de partida para enmarcar los resultados en los siguientes dos aspectos.

\section{Estadísticas de participación de las mujeres en la Olimpiada Estatal de Matemáticas en el nivel medio superior $(2018,2019)$}

El proceso de selección del equipo que representará a México en la Olimpiada Nacional de Matemáticas comienza regularmente en marzo, el adelanto o retraso de este depende de la autorización de la Subsecretaría de Educación Media Superior del estado y de la disponibilidad de recursos de parte de las instituciones que fungirán como sedes (Preparatorias, CBTIS, CBTA etc.). El estudiantado o el profesorado registra la participación de quienes se interesen en la página oficial del evento, por medio de un formulario. Sin embargo, este previo registro no impide que en la primera eliminatoria se presente alguien que no lo realizó por internet. La primera de las etapas es la municipal, para ello se procura que la mayoría de los municipios del estado cuente con una sede en la cual pueda llevarse a cabo la prueba. De esta se seleccionan los puntajes más altos para 
participar en una segunda etapa, la regional, que considera principalmente las 6 regiones geográficas en las que se divide el estado - Mante, Matamoros, Ciudad Madero, Nuevo Laredo, Ciudad Victoria, San Fernando. Finalmente, la etapa estatal se realiza en la capital que es Ciudad Victoria. Es importante mencionar que como una acción a futuro se permite la participación de estudiantado de secundaria. De este modo, para el siguiente año ya habrán tenido un acercamiento a este tipo de concursos (MATETAM, 2018).

En el 2019 el proceso de selección se vio retrasado debido a situaciones administrativas que involucran a las instituciones que son sede del concurso, razón por la cual el registro se llevó a cabo durante un periodo vacacional. Esta situación llevó a que el número de inscritos a la primera etapa en este año se redujo en casi una tercera parte en comparación con el del anterior. Pero, en cuanto a porcentajes de participación por sexo, el de mujeres aumentó (Gráfico 1). Una de nuestras hipótesis fue que dado que en la mayoría de los casos fue el estudiantado quien realizó su propio registro, una buena cantidad de estudiantes no espero a que sus docentes les convocarán y lo hicieron personalmente. En el año 2020 tuvimos una situación similar debido a las condiciones de las escuelas durante el confinamiento por pandemia. Esto sumado al acceso a una red de Internet redujo la participación casi a una tercera parte del año anterior.

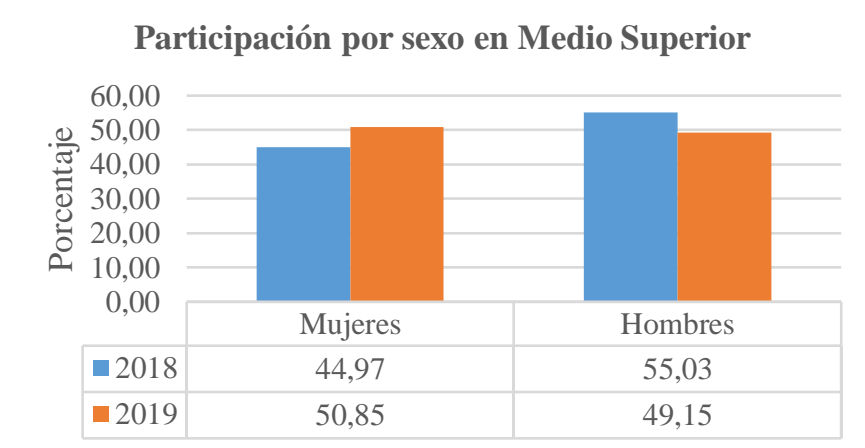

Gráfico 1: Porcentajes en la participación por sexo (Elaboración Propia)

Para el comité estatal es importante mantener un historial del número de veces que ha participado cada estudiante, esto con el objetivo de analizar su avance e historia dentro del concurso, pues entre sus principales finalistas han estado quienes tienen una larga trayectoria en el mismo.

Como es posible observar en los gráficos 2 y 3, el comportamiento es invertido. Mientras que en 2018 había una participación mayor por parte de los hombres desde la primera vez y se mantenía hasta la cuarta. En el 2019, observamos un porcentaje mayor 
de mujeres en su primera, tercera y cuarta participación. Y casi igual a la de los varones en la segunda. Significa, por lo tanto, que una buena cantidad de las jóvenes que se inscribieron, han participado en años anteriores y además entre quienes lo hacen por primera vez también supera al porcentaje de varones.

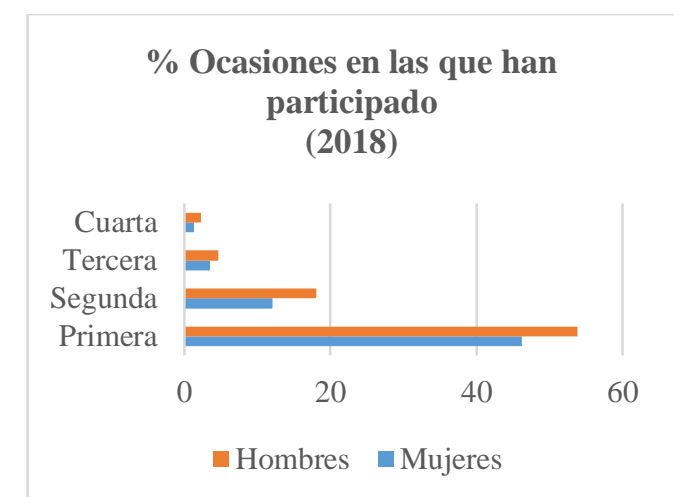

Gráfico 2 Permanencia 2018 (Elaboración Propia)

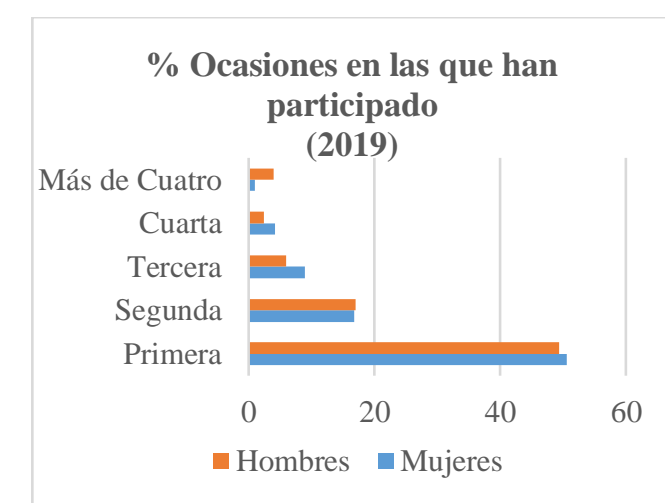

Gráfico 3 Permanencia 2019 (Elaboración Propia)

Como ya hemos mencionado, de este proceso de selección se obtiene un grupo de aproximadamente 30 estudiantes que trabajarán en una serie de entrenamientos, que tienen como objetivo encontrar a quienes formarán parte de los equipos del estado de Tamaulipas que participarán en la Olimpiada Nacional, pero también en el Concurso Regional del Noreste de la OMM. El proceso incluye además de los entrenamientos, una serie de exámenes selectivos mediante los cuales se elimina al estudiantado con los puntajes más bajos a través de varias rondas.

Esta preselección en 2018 estuvo compuesta por 32 estudiantes, de los cuales 24 fueron hombres y solo 8 mujeres. Para culminar con una selección de 6 hombres. En 2019 una de las primeras acciones que aplicamos fue que la preselección estaría compuesta por $50 \%$ mujeres y 50\% hombres. Así, quedo con por 17 mujeres y 18 hombres. La segunda acción afirmativa fue mantener a la preselección completa hasta que se aplicara la última de las pruebas de selección. El equipo final estuvo conformado por quienes sumaron, con las tres pruebas aplicadas, el puntaje más alto. Este estuvo conformado por 3 mujeres y 3 hombres. 


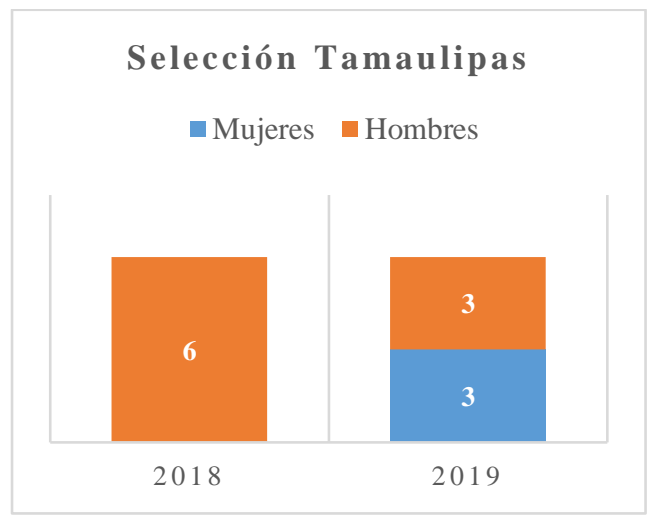

Gráfico 1 Sexo del estudiantado seleccionado (Elaboración Propia)

\section{Análisis de cuestionarios}

En la última etapa de la Olimpiada de Matemáticas en Tamaulipas se aplicó un cuestionario a las participantes mujeres y a sus acompañantes. Estos fueron contestados por 36 estudiantes y 19 acompañantes (14 madres, 3 padres y 2 asesores). Se toma esta etapa debido a que las jóvenes que llegaron a este punto son las que han pasado por dos eliminatorias y han mostrado un desempeño sobresaliente en todas ellas.

En los cuestionarios se analiza la interrelación de los tres elementos presentes en el modelo de desarrollo del talento: Matemáticas, talento y género. Según este, la relación que establezcan las jóvenes con la Matemática estará en función de las experiencias que vivan en su contexto - escuela, familia, concursos de olimpiada -; la concepción de talento en Matemáticas que se promueva en dichos espacios y por supuesto cómo esta relación está marcada por las atribuciones sociales que se depositan sobre ellas según su sexo de nacimiento. Por lo tanto, los cuestionarios están planteados con el objetivo de analizar cómo es la relación que hasta este momento han establecido con la Matemática a través de uno de sus principales entornos de socialización, la familia.

Los cuestionarios se enfocaron en analizar las acciones de acompañamiento en el desarrollo del potencial de sus hijas, así como sus expectativas profesionales: Apoyo en actividades escolares, actividades extraescolares, cercanía afectiva, máximo grado escolar esperado para ellas y el área en la que esperan se desempeñarán como profesionales.

\subsection{Padres y Madres}

La mayoría de las familias están compuestas por cuatro o cinco integrantes. En 
general muestran cercanía con el desarrollo académico de las jóvenes, están pendientes de sus necesidades educativas (intelectuales y materiales) y tiempos de estudio. A todas ellas suelen mostrarles su afecto y realizan actividades recreativas en familia. Todo esto indica el interés de las madres y los padres por apoyar el desarrollo del potencial de estas jóvenes. Sin embargo, al cuestionarles sobre sus expectativas profesionales, encontramos un contraste importante: 13 de los 17 familiares que contestaron el cuestionario expresan que esperan que ellas estudien un posgrado (5 maestría y 8 doctorado), los 4 restantes esperan que estudien solo licenciatura. El contraste lo encontramos en que ninguno de los 3 padres eligió el nivel doctorado, solo uno maestría o especialidad y 3 licenciatura universitaria o tecnológica. Si bien, la participación de los padres al contestar el cuestionario fue mínima, y dados los resultados de investigaciones anteriores eso suponíamos, ninguno de ellos espera que ellas alcancen el máximo nivel de estudios de entre los presentados.

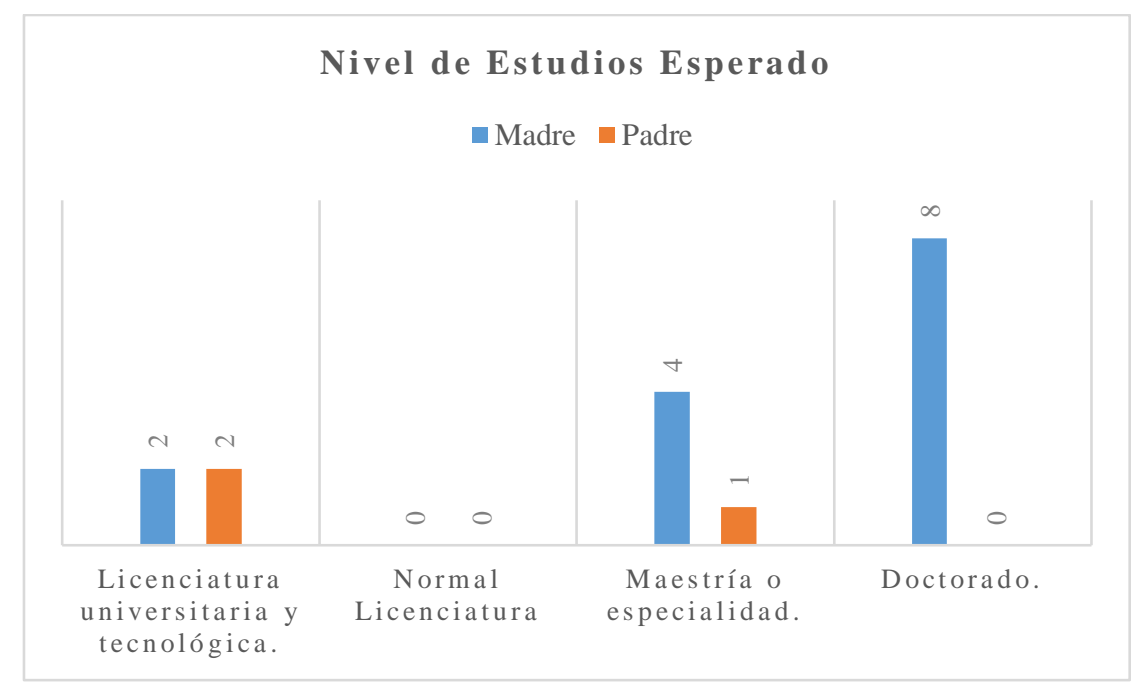

Gráfico 4: Nivel de estudios esperado por las madres y los padres (Elaboración Propria)

Como era de esperar, dado que ellas se encuentran en la última etapa del concurso, la mayoría de los padres y madres creen que ellas elegirán una carrera en el área Fisicomatemática, como segunda opción Químico-Biológicas. Ciencias Sociales y Humanidades tuvieron una mención. Artes no fue elegida.

\subsection{Las niñas}

Las estudiantes que llegaron a esta etapa del concurso tienen edades entre 12 y 16 años. Recordemos que las estudiantes de secundaria también son convocadas pues se 
espera que ellas comiencen a formarse en este tipo de actividades. Fueron las edades correspondientes a este nivel las que tuvieron una mayor participación (30 de 12-14 años). Solo 6 estudiantes tuvieron entre 15 y 16 años. Este también es un resultado esperado, pues como hemos mostrado antes, pocas mujeres suelen mantenerse a través de varias emisiones. Tal como lo habían mencionado las madres y los padres, estas jóvenes mantienen una relación cercana con sus familias: se sienten amadas, apoyadas y conversan sobre temas de su interés.

Respecto a las motivaciones por las cuáles ellas participan en la Olimpiada, se esperaría que en esta etapa del concurso su principal motivación estuviera relacionada con la Matemática misma. Sin embargo, solo la mitad de ellas (18) seleccionaron el "aprender muchas cosas" como una motivación. Por otro lado, 24 eligieron "es importante y lo necesito para el futuro", siendo esta una frase que forma parte del discurso de la mayoría de las personas cuando nos referimos a matemáticas.

En cuanto a su elección profesional, algunas de ellas están a uno o dos años de elegir un área del conocimiento en la cual desean desempeñarse y otras tienen aún algunos años para hacerlo. El área de conocimiento más mencionada fue la Fisicomatemática siendo 14 veces elegida, la segunda más popular fue la Químico-Biológica y sorpresivamente - contrario a la opinión de las padres y madres — encontramos a 5 jóvenes que eligieron artes. Los datos obtenidos muestran que más de la mitad de este grupo de jóvenes no elegiría una carrera estrechamente relacionada con Matemáticas $(59 \%)$.

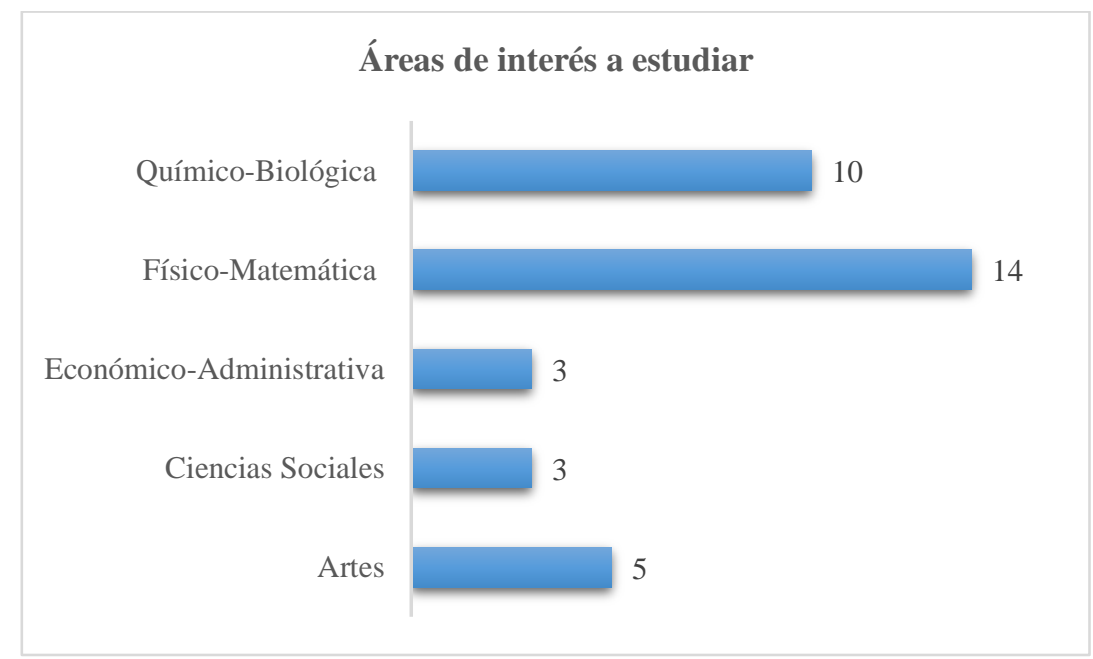

Gráfico 5: áreas de interés a estudiar por edad (Elaboración Propria)

Si bien artes no fue un área elegida por las madres y los padres si fue elegida las 
estudiantes y en mayor medida que otras áreas como Ciencias Sociales o EconómicoAdministrativas.

\subsection{Talleres y Entrenamientos de Olimpiada}

Durante dos meses, las 17 estudiantes de la preselección estatal participaron en actividades que se caracterizaron por su diversidad, es decir, además de formar parte de las sesiones de entrenamiento de olimpiadas realizaron otras actividades que tienen relación con la transversalidad de la Matemática hacia otras áreas de la Ciencia. El grupo de personas que trabajaron con las jóvenes en estas actividades estuvo compuesto en su mayoría por mujeres: 2 varones, 11 mujeres, más 1 asistente técnico (varón), tal como se muestra en la Tabla 2.

Tabla 2: Equipo académico de trabajo

\begin{tabular}{l|c|c}
\hline Tipo de Actividad & Mujeres & Hombres \\
\hline Conferencia & 3 & 0 \\
\hline Taller & 6 & 1 \\
\hline Entrenamiento de Olimpiada & 2 & 1 \\
\hline Total & 11 & 2 \\
\hline
\end{tabular}

Fuente: Elaboración Propria

Debido a que uno de los objetivos era mostrar una visión más amplia de la Ciencia, libre de estereotipos y sexismo, las conferencias y los talleres, además de contar con especialistas en matemática educativa de la UAT, se llevaron a cabo con el apoyo de investigadoras pertenecientes a la Red de Ciencia Tecnología y Género, quienes tienen una amplia experiencia en el acompañamiento de jóvenes mujeres en su formación como futuras profesionales en áreas STEM (BLAZQUEZ, GUERECA y MEZA, 2019). Como entrenadoras de olimpiadas se buscó a mujeres que estuvieran relacionadas con los procesos llevados a cabo por la OMM, pero también que estuviesen sensibilizadas con la importancia de integrar a más mujeres en estas actividades, pues tal como se ha documentado en varios estudios, metafóricamente como síndrome de la abeja reina (GARCÍA DE LEÓN, 2002), es posible que la posición de reconocimiento que han alcanzado les impida identificarse como parte de un grupo que es excluido de espacios de los que ellas forman parte.

En el caso de los talleres diseñados y llevados a cabo por especialista de la UAT, 
cada uno de los diseños didácticos se realizó bajo los principios de la teoría socioepistemológica, la cual da sustento al modelo de desarrollo del talento empleado en esta investigación. Sin embargo, su análisis requeriría de elementos mucho más amplios que lo que deseamos comunicar en este artículo.

Tanto los talleres como los entrenamientos de olimpiadas se caracterizaron por priorizar el trabajo colaborativo y destacar la participación de todas las integrantes del grupo. Características que en investigaciones previas se han identificado como aquellas que promueven el gusto e interés hacia las Matemáticas por parte de las mujeres.

Cuadro 1: Talleres, conferencias y entrenamientos de Olimpiada

\begin{tabular}{|l|l|}
\hline Taller & Temática \\
\hline Mujeres matemáticas & Matemáticas y género (Red Citeg/UAT) \\
\hline La magia de la luz & Física (Red Citeg) \\
\hline Dibuja la montaña & Matemáticas/Física (UAT) \\
\hline Representando la realidad en perspectiva & Matemáticas/Arte (UAT) \\
\hline Mi cuerpo perfectamente proporcional & Matemáticas/Anatomía (UAT) \\
\hline Frío o caliente & Matemáticas/Física (UAT) \\
\hline $\begin{array}{l}\text { Desarrollo de habilidades para Olimpiadas de } \\
\text { matemáticas }\end{array}$ & Matemáticas de Olimpiada (OMM) \\
\hline $\begin{array}{l}\text { Desarrollo de habilidades para Olimpiadas de } \\
\text { matemáticas }\end{array}$ & Matemáticas de Olimpiada (OMM) \\
\hline Conferencia & Temática \\
\hline $\begin{array}{l}\text { La trayectoria de una profesional de las } \\
\text { matemáticas }\end{array}$ & Matemática Educativa (Red Citeg/UAT) \\
\hline El papel de la mujer en las matemáticas & Género y Matemáticas (UAT) \\
\hline Distancias astronómicas & Astronomía (Red Citeg) \\
\hline
\end{tabular}

Fuente: Elaboración Propria

Siguiendo la propuesta de Marina Subirats (2010), 6 de los talleres fueron planeados solo para ellas.

\section{Discusión de resultados}

La literatura sobre desarrollo del talento considera que la identificación y atención temprana son claves para el futuro educativo del estudiantado, tanto de hombres como de mujeres (PRECKEL, PEKRUM y KLEINE, 2008; ELLISON y SWANSON, 2010; LEE y SRIRAMAN, 2012). Contrario a esto, investigaciones a nivel internacional han 
identificado que las experiencias tempranas de las mujeres jóvenes con una educación especializada en Matemáticas las alejan de, llegado el momento, elegir una carrera relacionada con esta área. Los gráficos 2 y 3 que muestra el número de ocasiones en las que el estudiantado ha participado en este tipo de concursos se relacionan con esto, pues la cantidad de estudiantes, tanto hombres como mujeres, se reduce al aumentar el número de participaciones. En el año 2018 la reducción en la participación de las mujeres fue más notoria que en 2019. Esto nos da píe para considerar al factor docente como un elemento crucial en la participación de estudiantes mujeres y hombres, dadas las condiciones en las que el registro se llevó a cabo.

Gran cantidad de investigaciones han dado evidencia de que los padres, las madres, el profesorado e incluso las mismas estudiantes consideran a la Matemática como un área masculina. Esta concepción puede llevar al profesorado a pensar en incluir o no, en su equipo o grupo de estudio a una estudiante mujer. Sin embargo, pareciera que al tener ellas la oportunidad de cultivar su gusto por las Matemáticas y de registrarse de manera individual al concurso, lo están haciendo. Muestra de ello podemos encontrar en las cifras del año 2020, las cuáles han sido obtenidas en condiciones especiales dado el confinamiento por la pandemia. Es por lo que no podemos considerar que estemos frente a un cambio significativo, pero es importante mencionarlo. En 2020 el registro y etapas de la Olimpiada Estatal se realizaron completamente en línea. La participación inicial fue de 647 personas, distribuido en 55.2\% mujeres y 44.8\% hombres. Después de dos rondas, la preselección quedó conformada por 45 estudiantes, 23 mujeres y 22 hombres. Cabe destacar que para esta preselección no se usó ningún tipo de acción afirmativa, como si se realizó para el proyecto en 2019. La selección estatal que participó en la Olimpiada Nacional estuvo conformada por 4 mujeres y 2 hombres, 3 de ellas y 2 de ellos ya habían formado parte de la selección el año anterior y la integrante nueva participó de las actividades de nuestro proyecto.

Los datos que presentamos muestran que la mayoría de estas jóvenes han descartado la elección de una carrera en un área estrechamente relacionada con Matemáticas. Lo cual indica que si bien, una intervención de este tipo en este momento puede dar resultados positivos, ya es demasiado tarde y el talento en Matemáticas que ellas poseen es muy probable que no sea aprovechado. Es por lo que las propuestas 
actuales consideran realizar programas de intervención a edades más tempranas (FLORES y GUEVARA, 2018).

El proyecto que describimos se fundamenta en el modelo teórico que hemos propuesto y en cómo los elementos Género, Matemáticas y Talento se correlacionan entre sí para que las niñas y jóvenes tengan las condiciones necesarias para desarrollar su potencial en matemáticas.

Con el objetivo de trastocar la concepción de talento en matemáticas que estas jóvenes han construido en relación con su contexto escolar y sociocultural. Planteamos una serie de actividades que buscan ampliar su visión sobre la matemática, desde una meramente escolar o de olimpiada a una que la considera una construcción humana que tiene relación con muchas áreas del conocimiento y en la que, además, las mujeres siempre han participado y actualmente tienen una presencia importante.

Subirats (2010), Mingo (2005) y Ursini y Ramírez (2017) han mostrado que el ambiente de aprendizaje que se genera en las aulas mixtas, tanto escolares como especiales no beneficia a las mujeres. Fue así como 6 de los talleres y todas las conferencias del proyecto estuvieron dirigidas solo a ellas. De esta forma, pudieron compartir con sus compañeras en un espacio donde las experiencias tanto individuales como grupales fueron valoradas, donde el diálogo y la cooperación fueron la basé de todas las actividades que allí se realizaron. Además, en todo momento se buscó destacar la presencia de mujeres que eligieron matemáticas, o áreas relacionadas como una opción profesional, tanto en los contenidos, como en las participantes del proyecto.

Un elemento importante es que estas jóvenes no se destacan únicamente en el campo de la Matemática. Identificamos que ellas tienen éxito en todas sus actividades académicas y que algunas participan de otros concursos y olimpiadas o se destacan en el dominio de otros idiomas. Así como una diversidad de actividades extraescolares que pueden incluir el arte, la música o los deportes. Esto contrasta con la idea tradicional que impera en la sociedad sobre las personas que sobresalen en matemáticas, la cual se refuerza en los diferentes medios de comunicación, televisión y cine. Esta es una situación reportada por otras investigaciones y estas han mostrado que finalmente las jóvenes se inclinarán por otras áreas del conocimiento en las que también se destacan.

Es importante mencionar que resultados significativos, producto de esta 
intervención y las sucesivas, solo podrán verse reflejados en el grupo de jóvenes de menor edad que formaron parte de las actividades.

\section{Referencias}

BLACK Laura; RADOVIC, Darinka; SALAS, Christian; WILLIAMS, Julian. Being a girl mathematician: diversity of positive mathematical identities in a secondary classroom. Journal for Research in Mathematics Education, Reston, v. 48, n. 4. P. 434464, jul./oct. 2017.

BLAZQUEZ, Norma; BUSTOS, Olga; FERNÁNDEZ, Lourdes. Saber y poder: vivencias de mujeres académicas. En: CONGRESO IBEROAMÉRICANO DE CIENCIA, TECNOLOGÍA Y GÉNERO, 9, 2012, Sevilla. Actas... Sevilla: Universidad de Sevilla, 2012, p. 1-22.

BLAZQUEZ, Norma; GUERECA, Raquel; MEZA, Lilia. Red Mexicana de Ciencia, Tecnología y Género: características y aportaciones. In. MEZA, Lilia; PONCE, Silvina. (Coord.) La brecha de género en Matemática, Computación y Ciencias Naturales: un abordaje desde América Latina. Ciudad de México: Sociedad Mexicana de Física, 2019, p. 183-192.

CANCHÉ, Erika; FARFÁN, Rosa Maria. El talento en Matemáticas desde una perspectiva sociocultural: un eje para el logro de la equidad educativa. La Matematica e la sua didattica, Bologna, v. 25, n. 2, p. 97-118. oct. 2017.

CANTORAL, Ricardo; MONTIEL, Gisela; REYES-GASPERINI, Daniela. Socioepistemología, Matemáticas y realidad. Revista Latinoamericana de Etnomatemática, San Juan de Pasto, v. 7, n. 3, p. 91-116, oct. 2014

COVARRUBIAS, Pedro; MARÍN, Rigoberto. Evaluación de la propuesta de intervención para estudiantes sobresalientes: caso Chihuahua México. Revista Electrónica Actualidades Investigativas en Educación, San José, v. 15, n. 3, p. 1-32, sep./dic. 2015.

DOMÍNGUEZ Pilar. Sobredotación, mujer y sociedad. Faisca: Revista de Altas Capacidades, Madrid, v. 9, n. 1, p. 3-34, ene. 2002.

ELLISON, Glen; SWANSON, Ashley. The gender gap in secondary school Mathematics and high achievement levels: evidence from the American Mathematics Competitions. Journal of Economic Perspectives, v. 24, n. 2, p. 109-128, mar. 2010.

FARFÁN, Rosa Maria; SIMÓN, María Guadalupe. La construcción social del conocimiento: el caso de género y Matemáticas. México: Gedisa, 2016.

FLORES, Raquel. Representaciones de género de profesores y profesoras de Matemática, y su incidencia en los resultados académicos de alumnos y alumnas. Revista Iberoamericana de Educación, Madrid, v. 1, n. 43, p. 103-118, ene. 2007.

FLORES, María; GUEVARA, Elsa. Educación científica de las niñas, vocaciones 
científicas e identidades femenias: experiencias de estudiantes universitarias. Actualidades Investigativas en Educación, San José, v. 18, n. 2, p. 1-31, may./aug. 2018.

GARCÍA DE LEÓN, María Antonia. Herederas y heridas: elites profesionales femeninas. Valencia: Cátedra, 2002.

GONZÁLEZ, Rosa María. De cómo las maestras llegaron a ser mayoría en las escuelas primarias de México, Distrito Federal. Finales del siglo XIX y principios del XX: un estudio de género. Revista Mexicana de Investigación Educativa, Ciudad de México, v. 14, n. 42, p. 747-785, may./aug. 2009.

LEE, Kyeong Hwa Lee; SRIRAMAN, Bharath. Gifted girls and non-mathematical aspirations: a longitudinal case study of two gifted Korean girls. Gifted Child Quarterly, v. 56, n.1, p. 3-14, feb. 2012.

MÁRQUEZ, Alejandro. A 15 años de PISA: resultados y polémicas. Perfiles Educativos, Ciudad de México, v. 39, n. 156, p. 3-15, abr./jun. 2017.

MARTÍNEZ, Uxune. Mujer, ciencia y discriminación: del efecto Mateo a Matilda. Blog Mujeres con Ciencia. Leiona: Universidad del País Vasco, 2014.

MATETAM. Informe Olimpiadas 2018 y Resultado Nacional OMM 2018. Disponible en http://www.matetam.com/noticias/2018/11/informe-olimpiadas-2018-y-resultadosnacional-omm-2018; acceso el 15 feb. 2020.

MINGO, Araceli. ¿Quién Mordió la manzana? Sexo, origen social y desempeño en la universidad. Ciudad de México: UNAM/Fondo de Cultura Económica, 2006.

OMM - Olimpiada Mexicana de Matemáticas de la Sociedad Matemática Mexicana. Estructura y lineamientos de la organización de la OMM. Disponible en https://www.ommenlinea.org/presentacion/lineamientos; acceso el 22 abr. 2021.

POBLETE, Rolando. Género y Educación: trayectorias de vida para ellos y ellas. Revista Latinoamericana de Educación Inclusiva, Santiago de Chile, v. 5 n. 1, p. 63-77, jul./dic. 2011.

PRECKEL, Franzis; GOETZ, Thomas; PEKRUN, Reinhard; KLEINE, Michael. Gender differences in gifted and average-ability students: comparing girls' and boys' achievement, self-concept, interest, and motivation in Mathematics. Gifted Child Quarterly, v. 52, n. 2, p. 146-159, abr. 2008.

MÉXICO. Secretaria de Educación Pública. Propuesta de intervención: atención educativa a alumnos y alumnas con aptitudes sobresalientes. Ciudad de México: SEP, 2006.

SIMÓN, María Guadalupe. Las aptitudes matemáticas de los estudiantes del programa Niñ@s Talento del Distrito Federal.2009.224f. Tesis (Maestría en Ciencias: Matemática Educativa) - Centro de Investigación y de Estudios Avanzados. Instituto Politécnico Nacional. Ciudad de México. 
SIMÓN, María Guadalupe. Transversalidad de género en la enseñanza de las Matemáticas. De Este Lado, Puebla, v. 3, n. 2, p. 17-26, jul./dic. 2018.

SIMÓN, María Guadalupe. Vertientes actuales de investigación en género y talento en Matemáticas. Revista Internacional de Ciencias Sociales y Humanidades, Ciudad Victoria, v. 26, n. 2, p-109-136, jul./dic. 2016.

SUBIRATS, Marina. ¿Coeducación o escuela segregada? Un viejo y persistente debate. Revista de Sociología de la Educación, v. 3, n. 1, p. 143-158, en./apr. 2010.

UNAM - Universidad Nacional Autónoma de México. Instituto de Matemáticas. Olimpiada Mexicana de Matemáticas. 2019. Disponible en https://youtu.be/8QxN7bREg2A; acceso el 9 jul. 2021.

URSINI, Sonia; RAMÍREZ, Martha P. Equidad, género y Matemáticas en la escuela mexicana. Revista Colombiana de Educación, Cundinamarca, n. 73, p. 213-234, jul./dic. 2017. 